\title{
A SUFFICIENT CONDITION FOR THE LOWER SEMICONTINUITY OF PARAMETRIC INTEGRALS
}

\author{
BY \\ EDWARD SILVERMAN $\left({ }^{1}\right)$
}

\begin{abstract}
We use simple convex functions and standard techniques in area theory to treat Morrey's extension of McShane's lower semicontinuity theorem for parametric integrals. This enables us to eliminate some technical hypotheses, simplify the proof and obtain a more general result.
\end{abstract}

We make use of "simple areas" to obtain a somewhat strengthened version of a result of McShane concerning the lower semicontinuity of parametric integrals ([1], [2, p. 360], [4], [5]). The essential idea of a simple area is that the convex functions in the appropriate exterior product space have supporting linear functionals which are simple. For the $k$-dim problem in $E^{k+1}$, this condition is automatically satisfied and hence does not appear in [1]. If the integrand is simple, or a suitable convex function of simple areas, then the version of McShane's Theorem obtained in this paper is exactly the same as the analogous theorem for Peano area, and the method of proof differs but slightly from that used in the elementary theory of that area.

All of the remaining references are to [3].

If $W$ is a real normed finite-dimensional vector space let $\wedge^{k} W$ be its $k$ th exterior product. If $p \in W^{k}$ let $p=\left(p_{1}, \ldots, p_{k}\right)$ and $\wedge p=p_{1} \wedge \cdots \wedge p_{k}$ where each $p_{i} \in W$. If $\alpha \in \bigwedge^{k} W$ and if $\alpha=\bigwedge p$ for some $p \in W^{k}$ then $\alpha$ is simple. Let $W_{1}$ be the closed unit ball in $W$ and let $W^{\prime}$ be the dual of $W$. If $q \in W^{\prime k}$ then $q=\left(q^{1}, \ldots, q^{k}\right)$ where each $q^{i} \in W^{\prime}$.

Let $E$ be Euclidean space with $k \leqq \operatorname{dim} E<\infty$. Let $T=\bigwedge^{k} E$ and $T^{\prime}=\left(\bigwedge^{k} E\right)^{\prime}$ $\approx \wedge^{k} E^{\prime}$. Let $S$ and $S^{\prime}$ be the simple elements in $T$ and $T^{\prime}$, respectively. Let $e_{i} \in R^{k}$ where $e_{i}^{j}=\delta_{i}^{j}$ for $i, j=1, \ldots, k$.

Let $\psi$ be a continuous nonnegative valued function on $E \times T$. Let $m(a)=\inf \psi_{a} \mid T_{1}$ and $M(a)=\sup \psi_{a} \mid T_{1}$. Evidently $m$ and $M$ are continuous. Suppose from now on that $\psi_{a}$ is convex and positively homogeneous of degree one for each $a \in E$. Let $A=\{a \in E \mid m(a)>0\}$ and let $\theta$ be defined on $T^{\prime} \times A$ by

$$
\theta(\zeta, a)=\max \{[\alpha, \zeta] \mid \psi(a, \alpha) \leqq 1\}
$$

Received by the editors March 15, 1971.

AMS 1970 subject classifications. Primary 49A50; Secondary 26A63, 28 A75.

Key words and phrases. Simple area, parametric integral.

(1) This research was supported in part by the National Science Foundation under Grant 6075-50-13955. 
so that

$$
\frac{\|\zeta\|}{M(a)} \leqq \theta(\zeta, a)=\max _{\alpha \neq 0} \frac{[\alpha, \zeta]}{\psi(a, \alpha)} \leqq \frac{\|\zeta\|}{m(a)}
$$

Let $\tau$ be defined on $T^{\prime} \times E$ by

$$
\begin{aligned}
\tau(\zeta, a) & =1 / \theta(\zeta, a) & & \text { if } \zeta \neq 0 \text { and } a \in A \\
& =0 & & \text { otherwise. }
\end{aligned}
$$

LEMMA 1. $\tau_{\zeta}$ is lower semicontinuous, and $\tau_{\zeta} \mid A$ is continuous.

Proof. We can suppose $\zeta \neq 0$. Let $K$ be a compact subset of $A$. It is sufficient to show that $\tau_{\zeta} \mid K$ is continuous. Let $m_{0}=\min m \mid K$ and $M_{0}=\max M \mid K$. Then $0<m_{0}$ $\leqq M_{0}<\infty$. Let $a, b \in K$. There exist $\alpha, \beta \in T_{1}$ such that $\tau(\zeta, a)=\psi(a, \alpha)[\alpha, \zeta]^{-1}$ and $\tau(\zeta, b)=\psi(b, \beta)[\beta, \zeta]^{-1}$. Let $\varepsilon>0$. There exists $\delta>0$ such that $|\psi(b, \gamma)-\psi(a, \gamma)|$ $\leqq \varepsilon\|\gamma\|$ for all $\gamma \in T$ provided $a, b \in K$ and $\|a-b\|<\delta$. Hence

$$
\begin{aligned}
\tau(\zeta, b) & \leqq \psi(b, \alpha)[\alpha, \zeta]^{-1}=\tau(\zeta, a) \psi(b, \alpha) \psi^{-1}(a, \alpha) \\
& \leqq \tau(\zeta, a)[\psi(a, \alpha)+\varepsilon] \psi^{-1}(a, \alpha) \leqq \tau(\zeta, a)+\tau(\zeta, a) \varepsilon m_{0}^{-1}
\end{aligned}
$$

so that $\tau(\zeta, b)-\tau(\zeta, a) \leqq M_{0}\|\zeta\|^{-1} \varepsilon m_{0}^{-1}$. We conclude that $\left|\tau_{\zeta}(c)-\tau_{\zeta}(d)\right| \leqq$ $M_{0}\|\zeta\|^{-1} m_{0}^{-1} \varepsilon$ if $c, d \in K$ and $\|c-d\|<\delta$.

Let $b \in E$ and $\beta \in T$. Since $\psi_{b}$ is convex there exists $\zeta \in T^{\prime}$ such that $[\beta, \zeta]$ $=\psi(b, \beta)$ and $\zeta \leqq \psi_{b}$. If for each $\alpha \in S, \psi_{b}(\alpha)=\sup \left\{[\alpha, \zeta] \mid \zeta \leqq \psi_{b}\right.$ and $\left.\zeta \in S^{\prime}\right\}$ then $\psi_{b}$ is simple. Thus, $\psi$ is simple if for each $b \in E$ and $\beta \in S$ there exists $\zeta \in S^{\prime}$ such that $\zeta \leqq \psi_{b}$ and $[\beta, \zeta]=\psi(b, \zeta)$.

We assume through Theorem 2 that $\psi$ is simple.

If $\eta \in E^{\prime k}$ let $\eta^{*} \in L\left(E, \boldsymbol{R}^{k}\right)$ be defined by $\eta^{*} b=\sum_{i}\left[b, \eta^{i}\right] e_{i}$.

If $T^{\prime} \in L\left(E^{\prime}, E^{\prime}\right)$ let $T^{\prime} \eta=\left(T^{\prime} \eta^{1}, \ldots, T^{\prime} \eta^{k}\right) \in E^{\prime k}$ and let $T \in L(E, E)$ be defined as usual by $[T b, \xi]=\left[b, T^{\prime} \xi\right]$ for all $b \in E$ and $\xi \in E^{\prime}$. Of course $\operatorname{det} T=\operatorname{det} T^{\prime}$. Furthermore $T\left(\eta^{*} b\right)=\sum_{i}\left[T b, \eta^{i}\right] e_{i}=\sum_{i}\left[b, T^{\prime} \eta^{i}\right] e_{i}=\left(T^{\prime} \eta\right)^{*} b$.

Let $Q$ be an open subset of oriented $\boldsymbol{R}^{k}$ and let $C$ be the space of continuous functions on $Q$ into $E$ with the topology of uniform convergence on compact subsets of $Q$. If $x \in C$ and $\eta \in E^{\prime k}$ then, by the preceding paragraphs, $\eta^{*} x$ is a flat map from $Q$ into $\boldsymbol{R}^{k}$. If $q \in \boldsymbol{R}^{k}$ and $V$ is a bounded domain whose closure $\bar{V}$ is contained in $Q$ then $K^{+}\left(q, \eta^{*} x, V\right)$ is the essential positive multiplicity with which $\eta^{*} x \mid V$ takes on the value $q\left[3\right.$, p. 155]. If $f(q, x)=K^{+}\left(q, \eta^{*} x, V\right)$ then $f$ is lower semicontinuous on $\boldsymbol{R}^{k} \times C$ with the product topology [3, pp. 155-163]. Furthermore, if $T$ is a sense-preserving homeomorphism of $\boldsymbol{R}^{k}$ onto itself, then

$$
K^{+}\left(T q, T\left(\eta^{*} x\right), V\right)=K^{+}\left(q, \eta^{*} x, V\right) .
$$

Finally, if $\left\{V_{n}\right\}$ is a sequence of pairwise disjoint domains in $V$, then

$$
\sum_{n} K^{+}\left(q, \eta^{*} x, V_{n}\right) \leqq K^{+}\left(q, \eta^{*} x, V\right),
$$

i.e. $K^{+}$is superadditive. 
Lemma 2. If $\xi, \eta \in E^{\prime k}$ and if $\bigwedge \xi=\wedge \eta$ then

$$
\int_{R^{k}} K^{+}\left(q, \xi^{*} x, V\right) d q=\int_{R^{k}} K^{+}\left(r, \eta^{*} x, V\right) d r .
$$

Proof. There exists $T^{\prime} \in L\left(E^{\prime}, E^{\prime}\right)$ with $\operatorname{det} T^{\prime}=1$ such that $\xi=T^{\prime} \eta$. Let $q=T r$. Since $T\left(\eta^{*} x\right)=\left(T^{\prime} \eta\right)^{*} x, K^{+}\left(r, \eta^{*} x, V\right)=K^{+}\left(q, \xi^{*} x, V\right)$, and the lemma follows.

If $\zeta \in S^{\prime}$ and $\zeta=\bigwedge \xi$ for some $\xi \in E^{\prime k}$, we can, by the lemma, define $\mathscr{K}^{+}(\zeta, x, V)$ $=\int_{R^{k}} K^{+}\left(q, \xi^{*} x, V\right) d q$.

Let $\mathscr{V}$ be the collection of bounded domains $V$ with $\bar{V} \subset Q$. If $x \in C, \zeta \in T^{\prime}$ and $V \in \mathscr{V}$ let $H(x, V, \zeta)=\min \{\tau(\zeta, x(p)) \mid p \in \bar{V}\}$.

Lemma 3. Let $g(x)=H(x, V, \zeta)$. Then $g$ is lower semicontinuous.

Proof. Fix $x$. We can suppose that $g(x)>0$. Thus $x(\bar{V}) \subset A$. Hence there exists $\delta>0$ such that $y(\bar{V}) \subset A$ if $\|x-y\|<\delta$. For such $y$,

$$
|g(x)-g(y)| \leqq \max _{p \in \nabla}|\tau(\zeta, x(p))-\tau(\zeta, y(p))| .
$$

Since $\tau_{\zeta}$ is continuous on $A$, the lemma follows.

Let $F(x, V, \zeta)=H(x, V, \zeta) \mathscr{K}^{+}(\zeta, x, V)$ with the understanding that $0 \cdot \infty=0$.

Lemma 4. Let $h(x)=F(x, V, \zeta)$. Then $h$ is lower semicontinuous on $C$.

Proof. For fixed $V$ and $\zeta$ both $H(x, V, \zeta)$ and $\mathscr{K}^{+}(\zeta, x, V)$ are nonnegative and lower semicontinuous. Hence so is their product.

Now let $Z$ be the collection of finite subsets of $\mathscr{V}$ whose distinct elements are disjoint. We define a Peano-type area $P_{\psi}$ on $C$ by

$$
P_{\psi}(x)=\sup _{\sigma \in Z} \sum_{V \in \sigma} \sup _{\zeta \in S^{\prime}} F(x, V, \zeta) .
$$

The following theorem is an immediate consequence of the definitions and Lemma 4.

THEOREM 1. $P_{\psi}$ is superadditive and lower semicontinuous.

If $\xi^{*} x$ is essentially of bounded variation or essentially absolutely continuous [3, pp. 249, 251] for all $\xi \in E^{\prime k}$ then we say that $x$ is $e B V$ or $x$ is $e A C$, respectively. If $x$ is $e B V$ then we define $J_{e}^{+}\left(\xi^{*} x\right)$ to be the Lebesgue derivative (with respect to cubes) of $\mathscr{K}^{+}(\zeta, x)$ where $\zeta=\wedge \xi$, and $J_{e}^{-}\left(\xi^{*} x\right)$ is the Lebesgue derivative of $\mathscr{K}^{+}(-\zeta, x)$. Thus $J_{e}^{+}\left(\xi^{*} x\right) \cdot J_{e}^{-}\left(\xi^{*} x\right)=0$ almost everywhere [3, pp. 257-258]. Furthermore, if $V \in \mathscr{V}$ then $\mathscr{K}^{+}(\zeta, x, V) \geqq \int_{V} J_{e}^{+}\left(\xi^{*} x\right)$ whenever $x$ is $e B V$ and the equality holds if $x$ is $e A C$ [3, pp. 250-251]. If $x$ is differentiable then $\wedge x^{\prime}=x_{1} \wedge \cdots$ $\wedge x_{k}$ and $\left[\wedge x^{\prime}, \zeta\right]=J\left(\zeta^{*} x\right)$ where $J$ is the ordinary Jacobian. If $x$ is $e B V$ we define $\left[\wedge x^{\prime}, \zeta\right]^{+}=J_{e}^{+}\left(\xi^{*} x\right)$ whenever the right-hand side exists, which is almost everywhere. If $x$ is differentiable then $\psi\left(x, \wedge x^{\prime}\right)=\sup \left\{\tau(\zeta, x)\left[\wedge x^{\prime}, \zeta\right] \mid \zeta \in S^{\prime}\right\}$. Since $\psi$ and $\tau$ are nonnegative, $\psi\left(x, \wedge x^{\prime}\right)=\sup \left\{\tau(\zeta, x)\left[\wedge x^{\prime}, \zeta\right]^{+} \mid \zeta \in S^{\prime}\right\}$. If $x$ is $e B V$ we use this last equation to define $\psi\left(x, \wedge x^{\prime}\right)$ and let $I_{\psi}(x)=\int_{Q} \psi\left(x, \wedge x^{\prime}\right)$. 
THEOREM 2. If $x$ is $e B V$ then $P_{\psi}(x) \geqq I_{\psi}(x)$ and the equality holds if $x$ is $e A C$.

Proof. If $\zeta \in S^{\prime}$ then $P_{\psi}(x \mid V) \geqq F(x, V, \zeta)$ for all $V \in \mathscr{V}$. Thus $D P_{\psi} \geqq$ $\tau(\zeta, x)\left[\wedge x^{\prime}, \zeta\right]^{+}$almost everywhere where $D P_{\psi}(x)$ is the Lebesgue derivative (with respect to cubes) of $P_{\psi}(x)$. Since $P_{\psi}$ is superadditive and the above inequality holds for all $\zeta$, we get $P_{\psi}(x \mid V) \geqq I_{\psi}(x \mid V)$ for all $V \in \mathscr{V}$. Hence $P_{\psi}(x) \geqq I_{\psi}(x)$. Now let $x$ be $e A C$. If $\zeta \in S^{\prime}$ then $\psi\left(x, \wedge x^{\prime}\right) \geqq \tau(\zeta, x)\left[\wedge x^{\prime}, \zeta\right]^{+}$. Hence $I_{\psi}(x \mid V) \geqq F(x, \zeta, V)$ for all $V \in \mathscr{V}$. Thus $I_{\psi}(x) \geqq P_{\psi}(x)$ if $x$ is $e A C$.

If $x$ and $y$ are in $R^{M}$, we write $x \leqq y$ if $x^{i} \leqq y^{i}$ for $i=1, \ldots, M$. Let

$$
\boldsymbol{R}_{+}^{M}=\left\{x \in \boldsymbol{R}^{M} \mid x \geqq 0\right\} .
$$

Similarly, let $\boldsymbol{R}_{M}^{+}=\left\{p \in \boldsymbol{R}_{M}=\left(\boldsymbol{R}^{M}\right)^{\prime} \mid p \geqq 0\right\}$.

Lemma 5. Let $f \in C\left(\boldsymbol{R}_{+}^{M}\right)$. Suppose that $f$ is positively homogeneous of degree one and convex with $f(x) \leqq f(y)$ if $x \leqq y$. If $x_{n} \in \boldsymbol{R}_{+}^{M}$, and if $x \leqq \lim _{n} \inf _{n}$ then

$$
f(x) \leqq \lim _{n} \inf f\left(x_{n}\right) .
$$

Proof. Let $P=\left\{p \in \boldsymbol{R}_{M}^{+} \mid p \leqq f\right\}$. It is not hard to see that

$$
f(u)=\sup \{[u, p] \mid p \in P\} .
$$

Thus

$$
\begin{aligned}
f(x) & =\sup [x, p] \leqq \sup \left[\liminf _{n} x_{n}, p\right] \\
& \leqq \sup \liminf _{n}\left[x_{n}, p\right]=\liminf _{n} \sup \left[x_{n} p\right]=\liminf _{n} f\left(x_{n}\right)
\end{aligned}
$$

where the suprema are taken over all $p \in P$.

Now let $\Phi$ be nonnegative and positively homogeneous of degree one on $\boldsymbol{R}_{+}^{M}$ such that $\Phi(x) \leqq \Phi(y)$ if $x \leqq y$ and $\Phi\left(\lim _{n} \inf _{n} x_{n}\right) \leqq \lim \inf _{n} \Phi\left(x_{n}\right)$ whenever $x_{n}$ is a sequence in $\boldsymbol{R}_{+}^{M}$. The functions described in the last lemma satisfy these conditions. Let $\psi_{1}, \ldots, \psi_{M}$ be simple on $E \times T$ and let $\phi(a, \alpha)=\Phi\left(\psi_{1}(a, \alpha), \ldots, \psi_{M}(a, \alpha)\right)$. (The idea of using some such $\phi$ is due to John Breckenridge.) If $\zeta=\left(\zeta^{1}, \ldots, \zeta^{M}\right) \in S^{\prime M}$, where each $\zeta^{i} \in S^{\prime}$, let

$$
F(x, V, \zeta)=\left(F_{1}\left(x, V, \zeta^{1}\right), \ldots, F_{M}\left(x, V, \zeta^{M}\right)\right)
$$

where $F_{i}\left(x, V, \zeta^{i}\right)$ is defined relative to $\psi_{i}$ as $F\left(x, V, \zeta^{i}\right)$ was defined relative to $\psi$. We define

$$
P_{\phi}(x)=\sup _{\sigma \in Z} \sum_{V \in \sigma} \sup _{\zeta \in S^{\prime} M} \phi(F(x, V, \zeta)) .
$$

The following theorem is immediate.

THEOREM 3. $P_{\phi}$ is superadditive and lower semicontinuous.

If $x$ is $e B V$ let $I_{\phi}(x)=\int_{Q} \phi\left(x, \Lambda x^{\prime}\right)$. 
THEOREM 4. If $x$ is $e B V$ then $P_{\phi}(x) \geqq I_{\phi}(x)$ and the equality holds if $x$ is $e A C$.

Proof. One first observes that, for fixed $x$ and $\zeta \in S^{\prime M}, \Phi(F(x, \cdot, \zeta))$ is superadditive. The proof of Theorem 2 then goes through virtually without change.

Let $x \in C$. Then $x$ is $e A C$ under a variety of conditions, in particular if $x$ is Lipschitzian and, if $k=2, x$ is $e A C$ if $x \in H_{2}^{\prime}$ [3, pp. 374-377, 436].

It is easy to see that there also exists a Lebesgue-type area $L_{\phi}$. Let

$$
L_{\phi}(x)=\liminf _{z \rightarrow x} I_{\phi}(z)
$$

where $z$ is quasilinear. (If $Q$ is not polyhedral, there is a slight additional complication which also arises for ordinary Lebesgue area.) It is easy to see that $L_{\phi}(x)$ $\geqq P_{\phi}(x)$ and the equality holds for sufficiently nice $x$.

\section{REFERENCES}

1. E. J. McShane, On the semicontinuity of double integrals in the calculus of variations, Ann. of Math. (2) 33 (1932), 460-484.

2. C. B. Morrey, Jr., Multiple integrals in the calculus of variations, Die Grundlehren der math. Wissenschaften, Band 130, Springer-Verlag, New York, 1966. MR 34 \#2380.

3. T. Radó and P. V. Reichelderfer, Continuous transformations in analysis. With an introduction to algebraic topology, Die Grundlehren der math. Wissenschaften, Band 75, SpringerVerlag, Berlin, 1955. MR 18, 115.

4. E. Silverman, A problem of least area, Pacific J. Math. 14 (1964), 309-331. MR 30 \#1229.

5. - Simple areas, Pacific J. Math. 15 (1965), 229-303. MR 31 \#1584.

Division of Mathematical Sciences, Purdue University, Lafayette, Indiana 47907 